UC-34

Reporting Date: August 1974 Issued: September 1974

\title{
A Multiple Time Scale Perturbation for Two Frequency Nonlinear Taylor Instability
}

by

George N. White 
This report was prepared as an account of work sponsored by the United States Govarnment. Neither the United States nor the United States Atomic Energy Commission, nor any of their employees, nor any of their contractors, subcontractors, or their employees, makes any warranty, express or implied, or assumes any legal liability or responsibility for the accuracy, completeness or usefulness of any information, apparatus, product or process disclosed, or represents that its use would not infringe privately owned rights.

In the interest of prompt distribution, this LAMS report was not edited by the Technical Information staff.

Printed in the United States of America. Arailable from Nationol Technical Information Service

U.S. Department of Commerce 5285 Port Royal Road

Springfield, Virginia 22151

Price: Printed Copy $\$ 4.00$ Microfiche $\$ 1.45$ 
A MULTIPLE TIME SCALE PERTURBATION FOR TWO FREQUENCY

NONLINEAP TAYLOK INSTABILITY

by

George N. Whit:?
NOTICE

was prepared as an account of work This seport was neited States Government. Neither the I'inted States nor the United States Atomic Energy Commission, nor any of their employees, nor any ot Comirnission, nor any ortors, subcontractors, or their employees. makes iny warranty, express or implied, or assacy, com. fegal liability or responsibility for the aren apparatus, pleteness or usef uiness of any informatents that its use product or procesa dissiused, or teptesents
would not infringe privately awned eights.

\section{ABSTRACT}

The nonlinear interaction of two Taylor unstable waves of different $i$ itial amplitude and frequency is studied by means of a multiple time scale perturbation. Unlike the single time scale expansion, the multiple scale treatment shows, explicitly, a dependence of the growth rate on the initial amplitudes and frequencies and confirms the early growth rate dependency found in numerical calculations (White, 1974).

\section{INTRODUCTION}

Most instabilities do not arise from single frequency, small amplitude perturbations. In this paper, plane nonlinear Taylor instability for two waves of different initial ampljtude and frequency is investigated by a multiple time scale perturbation. Viscosity, surface tension, and compressibility are not taken into account. The multiple scale expansion shows, explicitly, a growth rate dependence on the injtial anplitudes and frequencies not exhibited by a standard single time scale perturbation (White, 1973). The growth rate dependency found in the multiple suale expansion is consistent with the behavior of numerical calculations of the same problem (White, 1974).

The standard analysis was. straightforward extension of Ingraham's (1954) single frequency nonlinear perturbation to the two frequency case. As with many such expansions, the behavior of the solution, in particular its dependence on the parameters of the problem, is not clear. In fact a summation of the series would be needed to see what was going on. The numerical study was then made to find out what interaction ᄂffects were prominent. The most obvious result from tise calculations was long wave modulation of the short wave growth, even at early times. Since the effect appeared early, an appropriate perturbation should have a chance of representing the behavior. In particular, the multiple time scale expansion used by Nayfeh (1969) for the single frequency problem looked promising for the two frequency case. It will be seen that the multiple scale technique does show the modification of growth rates iy the initial. amplitudes and frequencies consistent with the results from the calculations.

II. THE DIFFERENTIAL EQUATIONS

The particular problem being considered is that of a plane, semi-infinite, inviscid, incompressible fluid without surface tension initially positioned above a vacuum. The initial interface elevation is given by

$$
n(x, 0)=n_{0} \cos k x+\eta_{m o} \cos m k x .
$$

The initial velocity is assumed to be zerc,

$$
n_{t}(x, 0)=0
$$

Under the action of gravity and in linear motion the interface position is the superposition of two waves $n(x, t)=\eta_{0} \cosh \sqrt{g k} t \cos k x+\eta_{\text {om }} \cosh \sqrt{m g k} t \cos m k x$

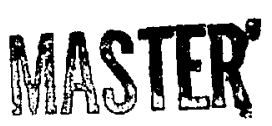


where $g$ is the acceleration of gravity. The nonlinear treatment $w i l l$ be seen to introduct factors multiplying the time $t$ and hence to modify the growth rates as well as to contribute higher order Fourier components.

If $x, y, \eta$ are made dimensionless by $k$, time by $\sqrt{\mathrm{gk}}$, and the velocity potential $\phi$ by $k \sqrt{\mathrm{gk}}$, the governing partial differential equation for $\phi$ is

$$
\phi_{x x}+\phi_{y y}=0
$$

in the region $-\infty<x<\infty, y \geqslant n, t \geqslant 0$. On the boundary $y=n$ where the pressure is zero Bernoulli's equation is

$$
-\phi_{t}+\frac{1}{2}\left[\phi_{x}^{2}+\phi_{y}^{2}\right]+\eta=0 \text {, }
$$

and the kinematic condition is

$$
\eta_{t}-\phi_{x} n_{x}+\phi_{y}=0
$$

The initial conditions are rewritten as

$$
\begin{aligned}
& n(x, 0)=\varepsilon \cos x+\alpha \varepsilon \cos m x \\
& n_{t}(x, 0)=0
\end{aligned}
$$

for $\varepsilon=k \eta_{0}, \alpha \varepsilon=k \eta_{m_{0}}$.

II1. THE PERTURBATION EXPANSION

The standard, second-order, perturbation in the long wave initial amplitude $E$ produces Fourier comcomponents $e^{ \pm i 2 x}, e^{ \pm i 2 m x}, e^{ \pm i(m \pm 1) x}$, in the two frequency problem. The intcrface $n$ is assumed therefore to be

$$
\begin{aligned}
n(x, t) & =\varepsilon\left(n_{1} e^{i x}+\bar{n}_{1} e^{-i x}\right)+\epsilon^{2}\left(n_{2} e^{i 2 x}+\bar{n}_{2} e^{-i 2 x}\right) \\
& +\varepsilon\left(n_{m} e^{i m x}+\bar{n}_{m} e^{-i m x}\right)+\varepsilon^{2}\left(n_{2 m} e^{i 2 m x}+\bar{n}_{2 m} e^{-i 2 m x}\right) \\
& +\varepsilon^{2}\left(n_{m+1} e^{i(m+1) x}+\bar{n}_{m+1} e^{-i(m+1) x}\right) \\
& +\varepsilon^{2}\left(n_{m-1} e^{i(m-1) x}+\bar{n}_{m-1} e^{-i(m-1) x}\right)+\cdots
\end{aligned}
$$

The boundary conditions $(2.5)$ and $(2.6)$ hold for $y=n$ and contain $\phi_{x}, \phi_{y}$, and $\phi_{t}$ evaluated at $y=n$.
If $\phi$ at $y=n$ is obtained by a power series expansion in $y, \phi$ needs only to satisfy Laplace's equation in the semi-infinite domain $-\infty<x<\infty, y \geqslant 0$, and therefore is taken to be

$$
\begin{aligned}
\phi(x, y, t) & =\varepsilon\left(\phi_{1} e^{i x}+\bar{\phi}_{1} e^{-i x}\right) e^{-y}+\varepsilon^{2}\left(\phi_{2} e^{-i 2 x}+\bar{\phi}_{2} e^{-i 2 x}\right) e^{-2 y} \\
& +\varepsilon\left(\phi_{m} e^{i m x}+\bar{\phi}_{m} e^{-i m x}\right) e^{-m y} \\
& +\varepsilon^{2}\left(\phi_{2 m} e^{i 2 m x}+\bar{\phi}_{2 m} e^{-i 2 m x}\right) e^{-2 m y} \\
& +\varepsilon^{2}\left(\phi_{m+1} e^{i(m+1) x}+\phi_{m+1} e^{-i(m+1) x}\right) e^{-(m+1) y} \\
& +\varepsilon^{2}\left(\phi_{m-1} e^{i(m-1) x}+\bar{\phi}_{m-1} e^{-i(m-1) x}\right) e^{-(m-1) y}+\ldots
\end{aligned}
$$

If the partial derivatives of $\eta$ and $\phi$ are obtained from these expansions and substituted into the boundary conditions, the following perturbation equations are found with terms up to third-order in $\varepsilon$ being rotuined

$$
\begin{aligned}
\dot{n}_{1}-\phi_{1} & =\varepsilon^{2}\left\{\bar{\phi}_{1} n_{2}-2 \bar{n}_{1} \phi_{2}-\frac{\bar{\phi}_{1} n_{1}^{2}}{2}+\phi_{1} n_{1} \bar{n}_{1}\right. \\
& -m \phi_{m} \bar{n}_{m-1}+m \bar{\phi}_{m} n_{m+1}+m^{2} \phi_{m} \bar{n}_{m} n_{1}-m^{2} \bar{\phi}_{m} n_{m} n_{1} \\
& \left.+(m-1) n_{m} \bar{\phi}_{m-1}-(m+1) \bar{n}_{m} \phi_{m+1}+\phi_{1} n_{m} \bar{n}_{m}\right\} \\
& \equiv \varepsilon^{2} \mathbf{f},
\end{aligned}
$$

$\dot{\phi}_{1}-n_{1}=\varepsilon^{2}\left\{\dot{\bar{\phi}}_{1} n_{2}+2 \bar{n}_{1} \dot{\phi}_{2}-\frac{\dot{\bar{\phi}}_{1} \eta_{1}^{2}}{2}-\dot{\phi}_{1} n_{1} \bar{n}_{1}-4 \phi_{1} \bar{\phi}_{1} n_{1}\right.$

$+4 \bar{\phi}_{1} \phi_{2}+m \dot{\phi}_{m} \bar{n}_{m-1}+\dot{m}_{m} n_{m+1}+(m-1) n_{m} \dot{\phi}_{m-1}$

$+(m+1) \bar{\eta}_{m} \dot{\phi}_{m+1}-\dot{\phi}_{1} n_{m} \bar{n}_{m}-m^{2} \dot{\phi}_{m} n_{1} \bar{n}_{m}-m^{2} \dot{\phi}_{m} n_{1} \eta_{m}$

$-4 m^{3} \phi_{m} \Phi_{m} n_{2}-2 m(m+1) \phi_{1} \Phi_{m} n_{m}$

$\left.+2 m(m-1) \phi_{m} \bar{\phi}_{m-1}+2 m(m+1) \bar{\phi}_{m} \phi_{m+1}\right\}=\varepsilon^{2} g$, 


$$
\begin{aligned}
& \dot{\eta}_{m}-m \phi_{m}=\varepsilon^{2} \mid m^{2} \bar{\phi}_{m} n_{2 m}-2 m^{2} \bar{n}_{m}{ }_{2 m}-m^{3} \frac{\phi_{m}}{2} n_{m}^{2}+m^{3} \phi_{m} n_{m} \bar{n}_{m} \\
& -m \phi_{1} n_{m-1}+m \bar{\phi}_{1} n_{m+1}+m^{3} \phi_{m} n_{1} \vec{n}_{1}+m \phi_{1} \bar{n}_{1} n_{m} \\
& -m \bar{\phi}_{1} n_{1} n_{m}-m(m-1) n_{1} \phi_{m-1}-m(m+1) \bar{n}_{1} \phi_{m+1} \mid \\
& \equiv \varepsilon^{2} f_{m} \\
& \dot{\phi}_{m}-n_{m}=\left.\varepsilon^{2}\right|_{m} \dot{\bar{\phi}}_{m} n_{2 m}+2 m \dot{\phi}_{2 m} \bar{\eta}_{m}-\frac{m^{2}}{2} \dot{\bar{\phi}}_{m} n_{m}^{2}-m^{2} \dot{\phi}_{m} n_{m} \bar{n}_{m} \\
& -m^{2} \dot{\phi}_{m} n_{1} \bar{n}_{i}+(m-1) n_{1} \dot{\phi}_{m-1}+(m+1) \bar{n}_{1} \dot{\phi}_{m+1} \\
& +\dot{\phi}_{1} n_{m-1}+\dot{\bar{\phi}}_{1} n_{m+1}-\dot{\phi}_{1} \bar{n}_{1} n_{m}-\dot{\bar{\phi}}_{1} n_{1} n_{m} \\
& +4 m^{2} \bar{\phi}_{m} \phi_{2 m}-4 m^{3} \phi_{m} \bar{\phi}_{m} \eta_{m}-2 m(m+1) \phi_{m} \bar{\phi}_{1} \eta_{1} \\
& +2(m+1) \bar{\phi}_{1} \phi_{m+1}-4 \phi_{1} \bar{\phi}_{1} n_{3 m} l=\varepsilon^{2} g_{m} \\
& \dot{\eta}_{2}-2 \phi_{2}=-2 \phi_{1} \eta_{1} \text {, } \\
& \dot{\phi}_{2}-n_{2}=\dot{\phi}_{1} n_{1} \text {, } \\
& \dot{n}_{2 m}-2 m \phi_{2 m}=-2 m^{2} \phi_{m} n_{m} \text {, } \\
& \dot{\phi}_{2 \mathfrak{m}}-n_{2 \mathfrak{m}}=m \dot{\phi}_{m} n_{\mathfrak{m}}, \\
& \dot{\eta}_{m+1}-(m+1) \phi_{m+1}=-(m+1) \phi_{1} n_{m}-m(m+1) \phi_{m} n_{1},
\end{aligned}
$$$$
\dot{\phi}_{m+1}-n_{m+1}=m \dot{\phi}_{1 n} n_{1}+\dot{\phi}_{1} n_{m}
$$$$
\dot{\eta}_{m-1}-(m-1) \phi_{m-1}=(m-1) \bar{\phi}_{1} n_{m}-m(m-1) \phi_{m} \bar{n}_{1} \text {, }
$$$$
\dot{\phi}_{m-1}-n_{m-1}=m \dot{\bar{\phi}}_{m} n_{1}+\dot{\phi}_{1} n_{m}+2 m \bar{\phi}_{1} \phi_{m}
$$

The conjugate quantities in these equations are needed for traveling wave solutions. For the stationary, unstable case under consideration, the quantities are real. The initial conditions for the displacement components are

$$
\begin{aligned}
& \eta_{1}(0)=1 / 2 \quad \dot{n}_{1}(0)=0 \\
& \eta_{m}(0)=\alpha / 2 \quad \dot{\eta}_{m}(0)=0 \\
& \eta_{2}(0)=\dot{\eta}_{2}(0)=0 \\
& \eta_{2 m}(0)=\dot{n}_{2 m}(0)=0 \\
& n_{m+1}(0)=\dot{n}_{m+1}(0)=0 \\
& \eta_{m-1}(0)=\dot{n}_{m-1}(0)=0 .
\end{aligned}
$$

If the equations (3.3) - (3.14) are solved to the second crder by standard perturbation, the inhomogeneous terms in (3.3) - (3.6) are not needed. In the multiple time scale expansion the third order terms are essential as will be seen.

The perturbation expansions (3.3) - (3.14) were checked by Wayne Fullerton of the Lo. lamos Scientific Laboratory using a program in sLTRAN, a language and system for algebraic manipulations.

IV. THE MULTIPLE TIME SCALE EXPANSION

The method of multiple time scale expansions is discussed with many examples and many variants by Nayfeh (1973). The application of the method to the two frequency Taylor problem is a simple extension of Nayfeh's (1969) treatment of the single frequency instubility.

The need for multiple scale and other related perturbation techniques such as averaging, coordinate stretching, matched asymptotic expansicns, etc. arises from the fact that conventional perturbations often produce expansions which are not uniformly convergent. Most such expansions are devised to extend the range of convergence and achieve this by introducing additional variables which are then chosen so that terms contributing to the nonuniformity are eliminated.

In the stability probleis under consideration, the multiple scales consist of

$$
T_{0}=t, T_{1}=\varepsilon t, T_{2}=\varepsilon^{2} t
$$


The time derivative must be replaced by

$$
\frac{d}{d t}=\frac{\partial}{\partial T_{0}}+E \frac{\partial}{\partial T_{1}}+\varepsilon^{2} \frac{\partial}{\partial T_{2}}
$$

The unknown functions $n_{1}, \phi_{1}$,etc. are regarded as functions of $\mathrm{T}_{0}, \mathrm{~T}_{1}, \mathrm{~T}_{2}$ instead of just $t$. These functions are also expanded in the form

$$
\begin{aligned}
& \eta_{1}=\eta_{10}+\varepsilon \eta_{11}+\varepsilon^{2} \eta_{12}+\cdots \\
& \phi_{1}=\phi_{10}+\varepsilon \phi_{11}+\varepsilon^{2} \phi_{12}+\cdots
\end{aligned}
$$

The two equations (3.3) and (3.4) expanded in this way give partial differential equations

$$
\begin{aligned}
& \frac{\partial \eta_{10}}{\partial \mathrm{T}_{0}}-\phi_{10}+\varepsilon\left\{\frac{\partial \eta_{10}}{\partial \mathrm{T}_{1}}+\frac{\partial \eta_{11}}{\partial \mathrm{T}_{0}}-\phi_{11}\right\} \\
& +\varepsilon^{2}\left\{\frac{\partial \eta_{10}}{\partial \mathrm{T}_{2}}+\frac{\partial \eta_{11}}{\partial \mathrm{T}_{1}}+\frac{\partial \eta_{12}}{\partial \mathrm{T}_{0}}-\phi_{12}\right\}=\varepsilon^{2} \mathrm{f}+\cdots \\
& \frac{\partial \phi_{10}}{\partial \mathrm{T}_{\mathrm{o}}}-\mathrm{n}_{10}+\varepsilon\left\{\frac{\partial \phi_{10}}{\partial \mathrm{T}_{1}}+\frac{\partial \phi_{11}}{\partial \mathrm{T}_{0}}-\eta_{11}\right\} \\
& +\varepsilon^{2}\left\{\frac{\partial \phi_{10}}{\partial \mathrm{T}_{2}}+\frac{\partial \phi_{11}}{\partial \mathrm{T}_{1}}+\frac{\partial \phi_{12}}{\partial \mathrm{T}_{0}}-n_{12}\right\}=\varepsilon^{2} \mathrm{~g}+\cdots
\end{aligned}
$$

Collection of coefficients of like powers in $\varepsilon$ provides equations

$$
\begin{aligned}
& \frac{\partial \eta_{10}}{\partial \mathrm{T}_{0}}-\phi_{10}=0, \frac{\partial \phi_{10}}{\partial \mathrm{T}_{0}}-n_{10}=0 \\
& \frac{\partial n_{10}}{\partial \mathrm{T}_{1}}+\frac{\partial \eta_{11}}{\partial \mathrm{T}_{0}}-\phi_{11}=0, \frac{\partial \phi_{10}}{\partial \mathrm{T}_{1}}+\frac{\partial \phi_{11}}{\partial \mathrm{T}_{0}}-n_{11}=0 \\
& \frac{\partial n_{10}}{\partial \mathrm{T}_{2}}+\frac{\partial n_{11}}{\partial \mathrm{T}_{1}}+\frac{\partial n_{12}}{\partial \mathrm{T}_{0}}-\phi_{12}=\mathrm{f} \\
& \frac{\partial \phi_{10}}{\partial \mathrm{T}_{2}}+\frac{\partial \phi_{11}}{\partial \mathrm{T}_{1}}+\frac{\partial \phi_{12}}{\partial \mathrm{T}_{0}}-n_{12}=\mathrm{g} .
\end{aligned}
$$

The solution to equations $(4.6)$ is

$$
\begin{aligned}
& \mathrm{n}_{10}\left(\mathrm{~T}_{0}, \mathrm{~T}_{1}, \mathrm{~T}_{2}\right)=\mathrm{A}_{10}\left(\mathrm{~T}_{1}, \mathrm{~T}_{2}\right) \mathrm{e}^{\mathrm{T}_{\mathrm{o}}}+\mathrm{B}_{10}\left(\mathrm{~T}_{1}, \mathrm{~T}_{2}\right) \mathrm{e}^{-\mathrm{T}_{0}} \\
& \phi_{10}\left(\mathrm{~T}_{0}, \mathrm{~T}_{1}, \mathrm{~T}_{2}\right)=\mathrm{A}_{10}\left(\mathrm{~T}_{1}, \mathrm{~T}_{2}\right) \mathrm{e}^{\mathrm{T} \mathrm{T}_{0}-\mathrm{B}_{10}\left(\mathrm{~T}_{1}, \mathrm{~T}_{2}\right) \mathrm{e}^{-\mathrm{T}_{0}} .}
\end{aligned}
$$

The next pair of equations (4.7) reduces to a single equation

$$
\frac{\partial^{2} n_{11}}{\partial T_{0}^{2}}-n_{11}=-2 \frac{\partial \phi_{10}}{\partial T_{1}}=-2\left(\frac{\partial A_{10}}{\partial T_{1}} e^{T_{0}}-\frac{\partial B_{10}}{\partial T_{1}} e^{-T_{0}}\right)
$$

If the derviatives $\partial \mathrm{A}_{10} / \partial \mathrm{T}_{1}, \partial \mathrm{B}_{10} / \partial \mathrm{T}_{1}$ are zero, the solution will contain no terms $T_{0} e^{ \pm T_{0}}$, the so-called secular term.j. The variable $T_{1}$ can be excluded from the other equations. Also, $n_{11}, \phi_{11}$ can be ignored because they provide no information additional to that from $n_{10}, \phi_{10}$.

When the problem is a periodic, the exponentiais in the solutions are complex and hence bounded. In that case the arguments take the form that $n_{11} / n_{10}$ should be bounded for all $T_{0}$ and similarly for $n_{12} / n_{10}$. Elimination of the terms containing $T_{0}$ accomplishes this and assures the convergence of the expansion so far as $T_{0}$ is concerned. With real exponentials, as in this unstable case, the convergence is valid only for $T_{0}$ sufficiently small. Even so, the elimination of the secular terns does change the character of the solution in the correct way. If this multiple time expansion is applied to all the equations, eight pairs of equations are obtained

$$
\frac{\partial \eta_{10}}{\partial \mathrm{T}_{0}}-\phi_{10}=0, \frac{\partial \phi_{10}}{\partial \mathrm{T}_{\mathrm{o}}}-\eta_{10}=0,
$$

$$
\begin{aligned}
\frac{\partial n_{12}}{\partial T_{o}} & -\phi_{12}=-\frac{\partial n_{10}}{\partial \bar{T}_{2}}+\bar{\phi}_{10} n_{20}-2 \bar{n}_{10} \phi_{20}-\frac{\bar{\phi}_{10}}{2} n_{10}^{2} \\
& +\phi_{10} n_{10} \bar{n}_{10}-m \phi_{m o} \bar{n}_{m-10}+m \bar{\phi}_{m o} n_{m+10}+m^{2} \dot{\psi}_{m o} \bar{n}_{m o} n_{10} \\
& -m^{2} \Phi_{m o} n_{m o} n_{10}+(m-1) n_{m o} \bar{\phi}_{m-10}-(m+1) \bar{n}_{m o} \Phi_{m+10} \\
& +\phi_{10} n_{m o} \bar{n}_{m o}
\end{aligned}
$$




$$
\begin{aligned}
& \frac{\partial \phi_{12}}{\partial \mathrm{T}_{0}}-n_{12}=-\frac{\partial \phi_{10}}{\partial \mathrm{T}_{0}}+\dot{\bar{\phi}}_{10} \mathrm{n}_{20}+2 \bar{n}_{10} \dot{\phi}_{20}-\frac{\dot{\bar{\phi}}_{10}}{2} n_{10}^{2} \\
& -\dot{\phi}_{10} \eta_{10} \bar{n}_{10}-4 \phi_{10} \bar{\phi}_{10} \eta_{10}+4 \bar{\phi}_{10} \phi_{20}+\mathrm{m}_{\mathrm{mo}} \bar{\eta}_{\mathrm{m}-10} \\
& +\dot{\bar{\phi}}_{m o} n_{m+10}+(m-1) n_{m 0} \dot{\bar{\phi}}_{m-1 a}+(m+1) \bar{n}_{m o} \dot{\phi}_{m+10} \\
& -\dot{\phi}_{10} n_{m o} \bar{n}_{m o}-m^{2} \dot{\phi}_{m o} n_{10} \bar{n}_{m o}-m^{2} \dot{\phi}_{m o} n_{10} n_{m o} \\
& -4 m^{3} \phi_{m o} \bar{\phi}_{m o} n_{10}-2 m(m+1) \phi_{10} \bar{\phi}_{m o} n_{m o} \\
& +2 m(m-1) \phi_{m 0} \bar{\phi}_{m-10}+2 m(m+1) \bar{\phi}_{m o} \phi_{m+10}, \\
& \frac{\partial n_{m o}}{\partial T_{0}}-m \phi_{m o}=0, \frac{\partial \phi_{m o}}{\partial T_{0}}-n_{m o}=0, \\
& \frac{\partial n_{m 2}}{\partial T_{0}}-m \phi_{m 2}=-\frac{\partial n_{m o}}{\partial T_{2}}+m^{2} \Phi_{m o} n_{2 m o}-2 m{ }^{2} \bar{n}_{m o} \phi_{2 m o} \\
& -m^{3} \frac{\bar{\phi}_{m o}}{2} n_{m o}^{2}+m^{3} \phi_{m o} n_{m o} \bar{n}_{m o}-m \phi 10^{n} m-10+m \Phi_{10} n_{m+10} \\
& +m^{3} \phi_{m o} n_{10} \bar{n}_{10}+m \phi_{10} \bar{n}_{10} n_{m o}-m \bar{\phi}_{10} n_{10} n_{m o} \\
& -m(m-1) n_{10} \phi_{m-10}-m(m+1) \bar{n}_{10} \phi_{m+10} \text {, }
\end{aligned}
$$$$
\frac{\partial \phi_{m 2}}{\partial T_{0}}-\eta_{m 2}=-\frac{\partial \phi_{m o}}{\partial T_{2}}+m \dot{\bar{\phi}}_{m o} \eta_{2 m o}+2 m \dot{\phi}_{2 m o} \bar{\eta}_{m o}
$$$$
-\frac{m^{2}}{2} \dot{\phi}_{m o} \eta_{m o}^{2}-m^{2} \dot{\phi}_{m o} \eta_{m o} \bar{\eta}_{m o}-m^{2} \dot{\phi}_{m o} \eta_{10} \bar{\eta}_{10}
$$$$
+(m-1) n_{10} \dot{\phi}_{m-10}+(m+1) \bar{n}_{10} \dot{\phi}_{m+10}+\dot{\phi}_{10} \eta_{m-10}
$$$$
+\dot{\bar{\phi}}_{10} \eta_{m+10}-\dot{\Phi}_{10} \bar{n}_{10} \eta_{m o}-\dot{\bar{\phi}}_{10} n_{10} n_{m o}+4 m^{2} \bar{\phi}_{m o} \phi_{2 m o}
$$$$
-4 m^{3} \phi_{m o} \bar{\phi}_{m o} n_{2 m o}-2 m(m+1) \phi_{m o} \bar{\phi}_{10} n_{10}
$$$$
+2(\mathrm{~m}+1) \bar{\phi}_{10} \phi_{\mathrm{m}+10}-4 \phi_{10} \bar{\phi}_{10} \mathrm{n}_{\mathrm{mo}} \text {. }
$$$$
\frac{\partial \eta_{20}}{\partial T_{0}}-2 \phi_{20}=-2 \phi_{10} \eta_{10} .
$$$$
\frac{\partial \Phi_{20}}{\partial T_{0}}-\eta_{20}=\dot{\phi}_{10} n_{10}
$$

$$
\begin{aligned}
& \frac{\partial \eta_{m-10}}{\partial T_{0}}-(m-1) \phi_{m-10}=(m-1) \bar{\phi}_{10} \eta_{m o}-m(m-1) \phi_{m o} \bar{\eta}_{10} \\
& \frac{\partial \phi_{m-10}}{\partial T_{o}}-\eta_{m-10}=m \dot{\phi}_{m o} \bar{n}_{10}+\dot{\phi}_{10} \eta_{m o}+2 m \bar{\phi}_{10} \phi_{m o} .
\end{aligned}
$$

$\frac{\partial \eta_{m+10}}{\partial T_{0}}-(m+1) \phi_{m+10}=-(m+1) \phi_{10} \eta_{m o}-m(m+1) \phi_{m o} \eta_{10}$

$$
\frac{\partial \phi_{m+10}}{\partial T_{0}}-n_{m+10}=m \dot{\phi}_{m o} n_{10}+\dot{\phi}_{10} n_{m o}
$$

All of these equations must be solved with the excepision of (4.12) and (4.14) which are only necessary for the elimination of secular terms. For completeness, solutions for all the remaining equations are collected now.

$$
\begin{aligned}
& \eta_{10}\left(T_{0}, T_{2}\right)=A_{10}\left(T_{2}\right) e^{T o}+B_{10}\left(T_{2}\right) e^{-T o}, \\
& \phi_{10}\left(T_{0}, T_{2}\right)=A_{10}\left(T_{2}\right) e^{T o}-B_{10}\left(T_{2}\right) e^{-T} 0 \\
& \eta_{m o}\left(T_{0}, T_{2}\right)=A_{m o}\left(T_{2}\right) e^{\sqrt{m} T_{0}+B_{m o}\left(T_{2}\right) e^{-\sqrt{m} T_{0}}} \\
& \phi_{m o}\left(T_{0}, T_{2}\right)=\left[A_{m o}\left(T_{2}\right) e^{\sqrt{m} T_{0}}-B_{m o}\left(T_{2}\right) e^{-\frac{1}{m} T_{0}}\right] / \sqrt{m}
\end{aligned}
$$

For simplicity the arguments in $\eta_{20}, \phi_{2 u}$, etc. and in coefficients $A_{10}, B_{10}, A_{\text {mo }}, B_{\text {mo }}$ are omitted. The coefficients of the higher order homogeneous solutions are not taken to be functions of $i_{2}$. Still higher order terms would be required to determine them, so they are considered constant. The higher order solutions are then 


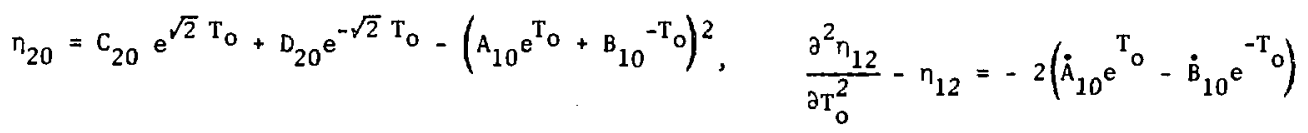

$$
\begin{aligned}
& \phi_{20}=\frac{1}{\sqrt{2}}\left(C_{20} e^{\sqrt{2} \mathrm{~T}_{\mathrm{o}}}-\mathrm{D}_{20} \mathrm{e}^{-\sqrt{2} \mathrm{~T}_{\mathrm{o}}}\right) \\
& +2 n_{10} n_{20}-5 \phi_{10}^{2} n_{10}^{2}-n_{10}^{3}+4 \phi_{10} \phi_{20} \\
& -6 m \phi 10 \phi_{m o} \eta_{m o}-2 n_{10} \eta_{m o}^{2}-2 m^{2}(2 m+1) \phi_{m o}^{2} \pi_{10} \\
& +2 m n_{m o} n_{m+10}+2 m(m+1) \phi_{m o} \phi_{m+10} \\
& \eta_{2 m o}=C_{2 m o} e^{\sqrt{2 m} T_{o}}+D_{2 m o} e^{-\sqrt{2 m} T_{o}} \\
& -m\left(A_{m o} e^{\sqrt{m} r_{0}}+B_{m o} e^{-\sqrt{m}} T_{0}\right)^{2}, \\
& +2 m(m-1) \phi_{m o} \phi_{m-10}+2(m-1) n_{m o} n_{m-10} \\
& \phi_{2 m o}=\frac{1}{\sqrt{2 m}}\left(c_{2 m o} e^{\sqrt{2 m}} T_{o}-D_{2 m o} e^{-\sqrt{2 m} T_{o}}\right) . \\
& \eta_{m+10}=C_{m+10} e^{\sqrt{m+1}} T_{o}+D_{m+10} e^{-\sqrt{m+1}} T_{0} \\
& -(m+1)\left(A_{10} e^{T_{0}}+B_{10} e^{-T_{0}}\right)\left(A_{m o} e^{e^{m} T_{0}}+B_{m o} e^{-\sqrt{m} T_{0}}\right) \\
& \phi_{m+10}=\frac{1}{\sqrt{m+1}}\left(C_{m+10} e^{\sqrt{m+1}} T_{o}-D_{m+10} e^{-\sqrt{m+1}} T_{o}\right) \text {, } \\
& \eta_{m-10}=C_{m-10} e^{\sqrt{m-1} T_{0}+D_{m-10} e^{-\sqrt{m-1}} T_{0}} \\
& +(m-1)\left(A_{10} e^{T_{o}}+B_{10} e^{-T_{o}}\right)\left(A_{m o} e^{\sqrt{m} T_{o}+B_{m o}} e^{-\sqrt{m} T_{0}}\right) \\
& \phi_{m-10}=\frac{1}{\sqrt{m-1}}\left(C_{m-10} e^{\sqrt{m-1}} T_{o}-D_{m-10} e^{-\sqrt{m-1}} T_{o}\right) \\
& +2 m\left(A_{10} e^{T_{o_{0}} B_{10}} e^{-T}\right)\left(A_{m o} e^{\sqrt{m} T_{o_{-}} B_{m o}} e^{-\sqrt{m} T_{0}}\right) . \\
& \frac{\dot{A}_{10}}{A_{10}}=-2 A_{10} B_{10}+2 m(m+2) A_{m o} B_{m o} \text {, } \\
& \frac{\dot{B}_{1 C}}{B_{10}}=2 A_{10} B_{10}-2 m(m+2) A_{m o} B_{m o} \text {, } \\
& \frac{\dot{A}_{\mathrm{mo}}}{\sqrt{\mathrm{m}} \mathrm{A}_{\mathrm{mo}}}=-2 \mathrm{~m}^{2} \mathrm{~A}_{\mathrm{mo}} \mathrm{B}_{\mathrm{mo}}-2(2 \mathrm{~m}+1) \mathrm{A}_{10^{B}} \mathrm{~B}_{1} \\
& \frac{\dot{B}_{m o}}{\sqrt{m} B_{m o}}=2 m^{2} A_{m o} B_{m o}+2(2 m+1) A_{10} B_{10}
\end{aligned}
$$


Aüdition of each pair of equations gives

$$
\begin{aligned}
& A_{10} B_{10}=k_{1}=\text { constant } \\
& A_{m o} B_{m o}=k_{m}=\text { constant }
\end{aligned}
$$

The solutions for the individual coefficients as functions of $\mathrm{T}_{2}$ are then

$$
\begin{aligned}
& A_{10}=A_{010} e^{\left[-2 k_{1}+2 m(m+2) k_{m}\right] T_{2},}, \\
& B_{10}=\frac{k_{1}}{A_{010}} e^{-\left[-2 k_{1}+2 m(m+2) k_{m}\right] T_{2}}, \\
& A_{\text {mo }}=A_{\text {omo }} e^{-\left[2 m^{2} k_{m}+2(2 m+1) k_{1}\right] \sqrt{m} T_{2}}, \\
& B_{\text {mo }}=\frac{k m}{A_{\text {omo }}} e^{\left[2 m^{2} k_{m}+2(2 m+1) k_{1}\right] \sqrt{m} T_{2},}
\end{aligned}
$$

If the boundary conditions (3.15) are applied to $\eta_{10}$ and $\eta_{\text {mo }}$ expressed in terms of (4.31), (4.32), the constants in the latter sets of equations are found to be

$$
\begin{aligned}
& A_{010}=B_{010}=1 / 4, \\
& A_{\text {omo }}=B_{\text {omo }}=\alpha / 4, \\
& k_{1}=1 / 16, \quad k_{m}=\alpha / 16
\end{aligned}
$$

With these values the second order displacement components, $n_{20}, \eta_{2 m o}, n_{m+10}, n_{m-10}$, are determined except for the coefficients in the homogeneous solutions. These coefficients are found by satisfying the zero initial displacement and velocity conditions of (3.15). The final expression for $\eta$ is given by where

$$
\begin{aligned}
& \sigma=\frac{1}{8}[-1+\alpha m(m+2)], \\
& \sigma_{m}=-\frac{1}{8}\left[m^{2} \alpha+(2 m+!)\right] .
\end{aligned}
$$

For the single frequency case (4.34) reduces to

$$
\begin{aligned}
n(x, t) & =\varepsilon \cosh \left(1-\frac{\varepsilon^{2}}{8}\right) t \cos x \\
& +\frac{\varepsilon^{2}}{2}\left[\cosh \sqrt{2} t-\cosh ^{2}\left(1-\frac{\varepsilon^{2}}{8}\right) t\right] \cos 2 x+\cdots
\end{aligned}
$$

the result obtained by Nayfeh (1969) when the depth of the fluid is infinite and surface tension is absent. For the standard two frequency expansion, the two quantities $\sigma$ and $\sigma_{m}$ are zero. The nonlinear initial amplitude contributions in $\sigma$ and $\sigma_{m}$ are respectively $-\frac{1}{8}$ an $-\frac{m^{2} \alpha}{8}$. The remaining terms in $\sigma$ and $\sigma_{m}, a n(m+2) / 8$, and $-(2 m+1) / 8$ are the result of interacion of the two waves.

\section{CONCLUSIONS}

From (4.34) with $\sigma=\sigma_{\mathrm{m}}=0$, the standard perturbation expansion, there is no evidence that initial amplitudes or frequencies have any effect on the growth rates. On the other hand, the full expansion (4.34) for the multiple time scale expansion does show the effects of $\varepsilon, \alpha$, and $m$ in the first and second order terms. The high frequency growth rate is reduced by tije factor $-(2 m+1) / 8$ whereas the low frequency rate is increased by $a m(m+2) / 8$. In the numerical calculations ( $\alpha=0.1, m=5, \varepsilon=0.1$ ) (White, 1974) the high frequency reduced rate was clear at early and late times. The increase in growth of the low frequency was not apparent even for the case $\alpha=1.0, \varepsilon=0.01$, probably because it is difficult to separate accurately the amplitudes

$$
\begin{aligned}
n(x, t) & =\varepsilon \cosh \left(1+\sigma \varepsilon^{2}\right) t \cos x \\
& +\frac{\varepsilon^{2}}{2}\left[\cosh \sqrt{2} t-\cosh ^{2}\left(1+\sigma \varepsilon^{2}\right) t\right] \cos 2 x \\
& +\alpha \varepsilon \cosh \left(1+\sigma_{m} \varepsilon^{2}\right) \sqrt{m} t \cos m x \\
& +m \frac{\alpha^{2} \varepsilon^{2}}{2}\left[\cosh \sqrt{2 m} t-\cosh ^{2}\left(1+\sigma_{m} \varepsilon^{2}\right) \sqrt{m} t\right] \cos 2 m x \\
& +\alpha \frac{(m+1)}{2} \varepsilon^{2}\left[\cosh \sqrt{m+1} t-\cosh \left(1+\sigma \varepsilon^{2}\right) t \cosh \left(1+\sigma_{m} \varepsilon^{2}\right) \sqrt{m} t\right] \cos (m+1) x \\
& +\alpha \frac{(m-1)}{2} \varepsilon^{2}\left[-\cosh \sqrt{m-1} t+\cosh \left(1+\sigma \varepsilon^{2}\right) t \cosh \left(1+\sigma_{m} \varepsilon^{2}\right) / m t \cos (m-1) x+0\left(\varepsilon^{3}\right)\right.
\end{aligned}
$$


of the two waves in that case. For $a=0, l$, of course, the low frequency is not affected as much as the high frequency.

While the removing of secular terms accomplishes the objective of revealing a growth dependent on initial amplitudes and frequencies, the validity of the solution is obviously not extended significantly in time. Such an extension is not to be expected when the secular terms involve terms with $t$ times an exponential in $t$ as occurs with these unstable problems. For traveling waves, the removal of the secular terms eliminates the time growth and a significant improvement in representation of the solution is achieved. In the single frequency case for $m \neq 1$ and in the two frequency case, convergence also is not uniform in the frequency $\mathrm{m}$. This shows up in the growth rates $\left(1+\sigma \varepsilon^{2}\right),\left(1+\sigma \varepsilon^{2}\right)$ as well.

It appears that multiple scale expansions, or other techniques such as averaging, coordinate stretching, or their modifications do not at this time offer the hope of extending the validity of the Taylor instability solution to late times with constant bubble velocity and free fall spike growth. Some approach fundamentally different from these perturbation methods is needed if large, nonlinear motions are to be followed.

\section{REFERENCES}

1. G. N. White, "Two Frequency Nonlinear Taylor Instability", Los Alamos Scientific Laboratory report LA-5575-MS (1974).

2. G. N. White, "Mode Mixing in Nonlinear Taylor Instability", Los Alamos Scientific Laboratory internal document (1973).

3. R. L. Ingraham, "Taylor Instability of the Interface Between Superposed Fluids-Solution by Successive Approy mations", Proc. Phys. Soc. (London) B67, 748 (1954).

4. A. H. Nayfeh, "On the Non1inear Lamb-Taylor Instability", J. Fluid Mech. 38, Part 3, 619 (1969).

5. A. H. Nayfeh, "Perturbation Methods", John Wiley \& Sons, New York (1973). 